\title{
Rice OsHOL1 and OsHOL2 proteins have S-adenosyl-L- methionine-dependent methyltransferase activities toward iodide ions
}

\author{
Yuko Takekawa, Tatsuo Nakamura* \\ Graduate School of Environment and Information Sciences, Yokohama National University, Yokohama, Kanagawa 240-8501, \\ Japan \\ *E-mail: t-nakamu@ynu.ac.jpＴel \& Fax: +81-45-339-4416
}

Received January 9, 2012; accepted February 7, 2012 (Edited by T. Demura)

\begin{abstract}
Food plants are important sources of iodine, an essential nutrient required for the synthesis of thyroid hormone in humans. Understanding iodine metabolism in plants is important for tackling iodine deficiency, a serious micronutrient deficiency in the world. Elucidation of iodine metabolism in plants is also important for understanding biogeochemical iodine cycling because plants absorb iodine from the soil and emit considerable amounts of gaseous iodine compounds into the atmosphere. HAMLESS TO OZONE LAYER (HOL) family proteins found in plants have been reported to synthesize methyl halides, including methyl iodide, from halide ions and S-adenosyl-L-methionine (SAM). Here, we report the kinetic analysis of two rice HOL proteins, named OsHOL1 and OsHOL2. Recombinant OsHOL1 and OsHOL2 proteins synthesized methyl iodide from an iodide ion and SAM. Kinetic analyses of the recombinant proteins showed that methyltransferase activities toward iodide ions were highest among the examined substrates-bromide, chloride, and thiocyanate ions. These results suggested that OsHOL1 and OsHOL2 are involved in iodine metabolism in rice and contribute to methyl iodide emissions from rice.
\end{abstract}

Key words: HOL protein, iodine, kinetics, Oryza sativa, S-adenosyl-L-methionine-dependent methyltransferase activity.

Iodine is an essential nutrient for humans, and iodine deficiency has many adverse effects on growth and development, including thyroid enlargement (goiter) and severe mental deficiency (Zimmermann et al. 2008). According to an estimation by WHO, approximately 2 billion people in the world have insufficient intake of iodine (de Benoist et al. 2008). Iodine deficiency disorders are among the most important micronutrient deficiencies, together with vitamin $\mathrm{A}$ and iron deficiencies (Mayer et al. 2008). Iodized salt is the most effective way to prevent iodine deficiency because most people consume salt daily. Although iodized salt is widely available, various types of social and technical problems hamper the availability of iodine through salt, especially in developing countries. Therefore, food plants that people eat everyday are still important iodine sources. Although elucidation of iodine metabolism in plants is necessary for the development of biofortified plants rich in iodine, knowledge of plant iodine metabolism is limited.

Methyl iodide emissions have been reported in various plant species (Saini et al. 1995). A genetic analysis of
Arabidopsis T-DNA insertion mutants clarified that the HARMLESS TO OZONE LAYER gene (HOL; AtHOL1 in our study) is involved in the emission of methyl iodide as well as methyl chloride and methyl bromide, which contribute to the destruction of the stratospheric ozone layer (Rhew et al. 2003). Arabidopsis possesses two further HOL paralogs, AtHOL2 and AtHOL3 (Nagatoshi and Nakamura 2007). Biochemical analyses of the three AtHOL proteins showed that they have SAMdependent methyltransferase activities toward iodide ions as well as chloride, thiocyanate, and hydrosulfide ions (Nagatoshi and Nakamura 2009). Plant proteins in which amino acid sequences showed high similarities to Arabidopsis HOL were classified as HOL family proteins, including independently characterized methyl chloride transferase (MCT) (Ni and Hager 1998, 1999), thiol methyltransferase (TMT) (Attieh et al. 1995, 2000, 2002), and halide/thiol methyltransferase (HTMT) (Itoh et al. 2009). MCT, TMT, and HTMT proteins are also shown to possess SAM-dependent methyltransferase activity toward not only iodide ions, but also toward ions such as chloride, bromide, thiocyanate, and hydrosulfide ( $\mathrm{Ni}$ and

Abbreviations: GC-MS, Gas chromatography-mass spectrometry; HPLC, High performance liquid chromatography; MALDI-TOF-MS, Matrixassisted laser desorption/ionization time-of-flight mass spectrometry; SAH, S-Adenosyl-L-homocysteine; SAM, S-Adenosyl-L-methionine; SDSPAGE, Sodium dodecyl sulfate-polyacrylamide gel electrophoresis.

This article can be found at http://www.jspcmb.jp/

Published online March 20, 2012 
Hager 1999; Attieh et al. 1995, 2000; Itoh et al. 2009). All kinetic analyses of plant HOL family proteins reported to date have been performed using proteins from Brassicales plants. A physiological substrate of AtHOL1 protein was suggested to be a thiocyanate ion produced by the catabolism of indole glucosinolates, which are characteristic secondary metabolites among Brassicales plants (Midorikawa et al. 2009; Nagatoshi and Nakamura 2009). These results suggested that Brassicales plants have HOL proteins that play a physiological role specific to Brassicales plants. In this paper, we focused on two rice HOL proteins. Because rice is one of the most important crops in the world, and rice paddies cover approximately $1 \%$ of the global land area (FAOSTAT 2010; see http:// faostat.fao.org/site/567/default.aspx\#ancor), we examined the possibility of involvement of the rice HOL proteins in iodine metabolism.

We have previously identified two rice $H O L$ genes, OsHOL1 and OsHOL2, using the amino acid sequence of AtHOL1 in a BLAST search of the Oryza sativa genome (Nagatoshi and Nakamura 2009). The length of OsHOL1 full-length cDNA (accession No. CT835256) was 741 bp encoding an open reading frame (ORF) of 246 amino acids. The predicted protein was calculated to have a molecular mass of $26.2 \mathrm{kDa}$. There were two full-length cDNAs (accession Nos. AK061306 and AK101884) for OsHOL2 with different transcription start sites. Two gene models (Os06g0153900-01, Os06g0153900-02) were proposed in the Rice Annotation Project Database (RAP-DB). In this paper, we designated the gene model (Os06g0153900-01) as OsHOL2, for which the $\mathrm{N}$-terminus of the ORF was 16 amino acids longer than that of the gene model (Os06g0153900-02). The length of OsHOL2 full-length cDNA (accession No. AK061306) was 753 bp encoding an ORF of 250 amino acids. The predicted protein was calculated to have a molecular mass of $27.3 \mathrm{kDa}$. Figure $1 \mathrm{~A}$ shows an alignment of amino acid sequences of HOL proteins of Arabidopsis (AtHOL1, AtHOL2, and AtHOL3) and rice (OsHOL1 and OsHOL2). The alignment indicated that OsHOL2 has a longer $\mathrm{N}$-terminal sequence than the other HOL proteins. Prediction of subcellular localization of OsHOL2 using the WoLF PSORT program (Horton et al. 2007) suggested that OsHOL2 may have an N-terminal transit peptide for chloroplast localization.

A phylogenetic tree was constructed using amino acid sequences of HOL family proteins from various land plants (Figure 1B). The tree indicated that HOL proteins are widespread among land plants and reflect the genealogy of the derived plant species, except for Brassicales plants, in which the HOL proteins were grouped into two clusters, Cluster I and Cluster II. Arabidopsis AtHOL1 in Cluster I was involved in the metabolism of indole glucosinolates, which are characteristic secondary compounds in Brassicales plants (Nagatoshi and Nakamura 2009). These results suggested that HOL proteins in Cluster I play a role specific among Brassicales plants. In the tree, HOL proteins in Cluster II (Brassicales plants), Cluster III (non-Brassicales dicotyledonous plants), and Cluster IV (monocotyledonous plants) were located near each other, which implies that these HOL proteins have a common physiological role in land plants (Nagatoshi and Nakamura 2009). HOL homologous proteins were also found in algae (Ohsawa et al. 2001; Toda and Itoh 2011) and fungi (Saxena et al. 1998) in addition to the plant HOL proteins. OsHOL1 and OsHOL2 belong to Cluster IV, together with other HOL proteins of monocotyledonous plants. OsHOL2 was more similar to HOL proteins of the other monocots than OsHOL1. The Rice Expression Profile Database (RiceXPro; http:// ricexpro.dna.affrc.go.jp) (Sato et al. 2010) showed that OsHOL1 is expressed mainly in the leaf blade, whereas OsHOL2 is expressed in the whole plant at lower levels than OsHOL1 (data not shown), implying their different physiological roles.

Whereas methyltransferase activity toward iodide ions had been reported in crude extract prepared from rice tissues (Itoh et al. 2009), it was not examined whether OsHOL1 and OsHOL2 possess SAMdependent methyltransferase activities toward iodide ions. Therefore, we first examined whether OsHOL1 and OsHOL2 have the activity toward iodide ions as well as bromide, chloride, and thiocyanate ions. For this purpose, recombinant OsHOL1 and OsHOL2 were first prepared. cDNA was synthesized from total RNA purified from shoot tissues of 11-day-grown rice (Oryza sativa L. ssp. japonica cv. Nipponbare). Because OsHOL2 was postulated to have an $\mathrm{N}$-terminal presequence, the ORF of a gene model (Os06t0153900-02) lacking 16 amino acids at the $\mathrm{N}$-terminus was used to express a recombinant OsHOL2 protein in Escherichia coli. cDNA fragments containing OsHOL1 and OsHOL2 were PCR-amplified using cDNA synthesized as a template, DNA polymerase KOD-plus (TOYOBO), and the outer primers for the first PCR ( $5^{\prime}$-ACTCGAGGC CGGGGCACAT- $3^{\prime}$ and $5^{\prime}$-GTCACCCGCACATGT CTCT-3' for OsHOL1, 5'-TATTATTACGAGCCG

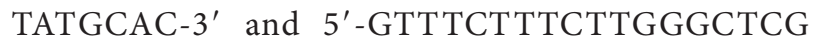
C-3' for OsHOL2). The second PCR was performed using the inner primers ( $5^{\prime}$-ATGGCGTCGGCGATC GT-3' and 5'-CACTCCTTGTTCGCAGCATA-3' for OsHOL1, 5' -ATGAGCTCGTCGGCGGC- ${ }^{\prime}$ and $5^{\prime}$ TAAAGAAGGGATGCAGCGTT-3' for OsHOL2). Each of the cDNA fragments was cloned in-frame with an N-terminal glutathione S-transferase (GST) tag into an expression vector pDEST15-T that was modified to possess a thrombin recognition site at the C-terminus of GST in pDEST15 (Invitrogen) (Nagatoshi and Nakamura 2007). The recombinant proteins digested with thrombin 
A

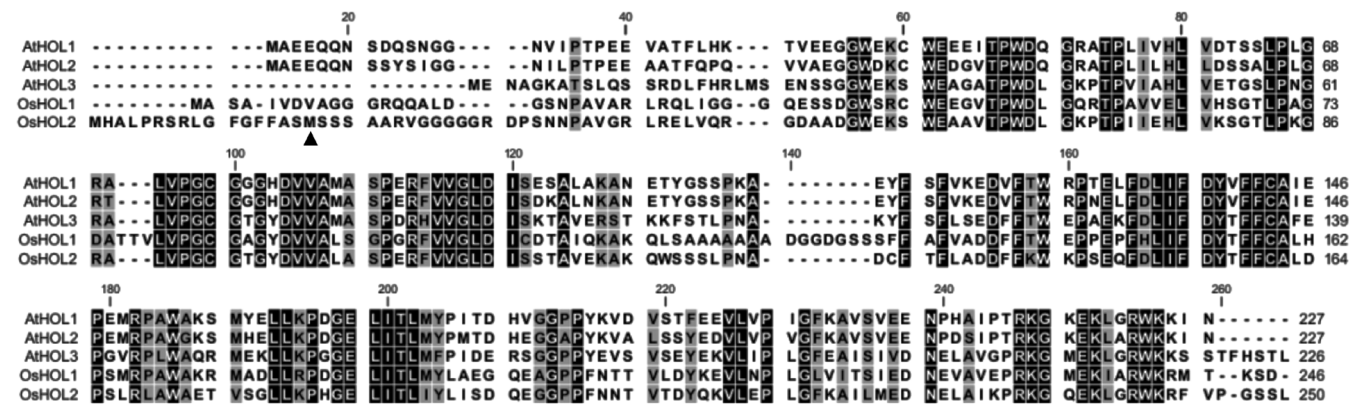

B

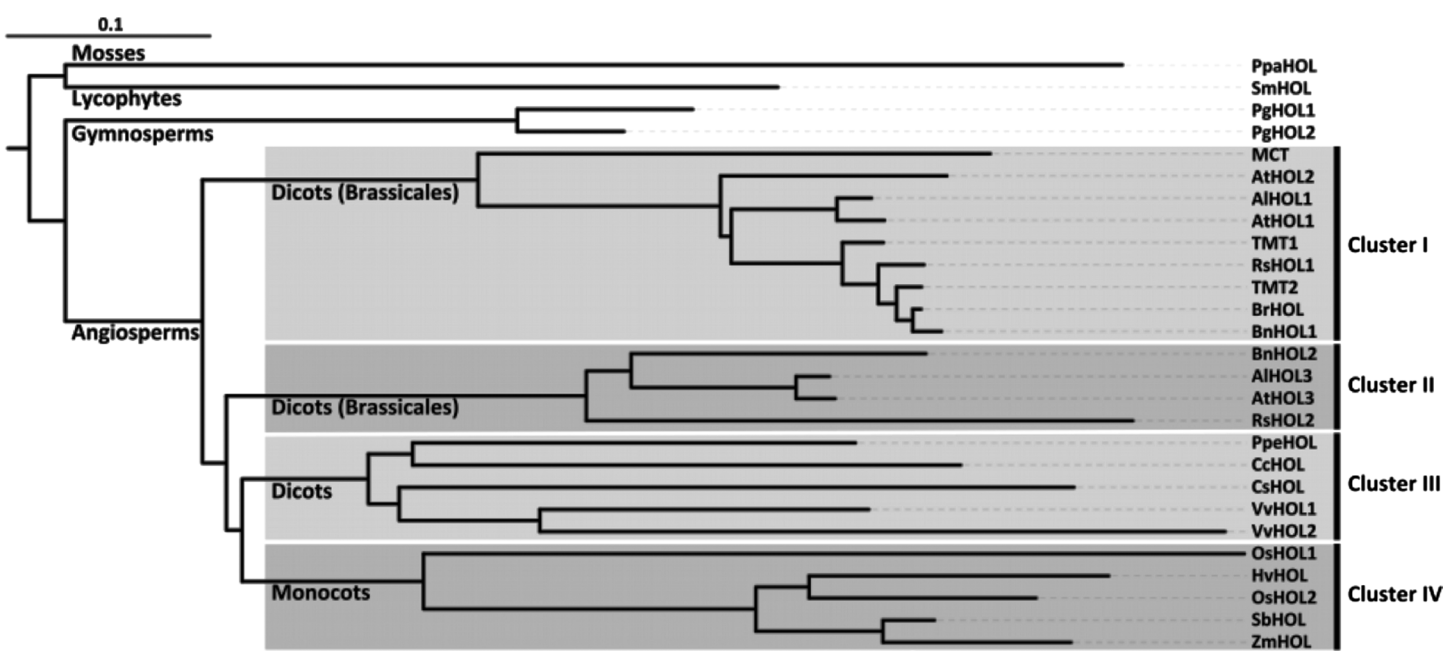

Figure 1. Properties of $H O L$ genes. (A) Multiple alignments of rice and Arabidopsis HOL proteins. Multiple alignments of the amino acid sequences of OsHOL1, OsHOL2, AtHOL1, AtHOL2, and AtHOL3 were built using Kalign (Lassmann and Sonnhammer 2005). Residues with black and gray background indicate identical residues among five and four sequences, respectively. An arrowhead indicates the first methionine of the amino acid sequence deduced from another OsHOL2 full-length cDNA (AK101884). (B) A molecular phylogenetic tree of HOL family proteins in plants. The amino acid sequences were aligned using the Kalign program, and the phylogenetic tree was built using the neighbor-joining method. Bar $=0.1$ amino acid substitutions/site. Sequences from Arabidopsis lyrata (AlHOL1 and AlHOL3: 483509 and 483510, respectively), A. thaliana (AtHOL1, AtHOL2, and AtHOL3: AT2G43910.1, AT2G43920.1, and AT2G43940.1, respectively), Batis maritima (MCT: AAD26120), Brassica napus (BnHOL1 and BnHOL2: TA24304_3708 and EV084135, respectively), Brassica oleracea (TMT1 and TMT2: AAK69760 and AAK69761, respectively), Brassica rapa (BrHOL: ABL86248), Coffea canephora (CcHOL: TA6982_49390), Cucumis sativus (CsHOL, Cucsa.169960), Hordeum vulgare (HvHOL: TA43367_4513), Oryza sativa (OsHOL1 and OsHOL2: Os03t0843800-00 and Os06t0153900-01, respectively), Physcomitrella patens (PpaHOL: TA15859_3218), Picea glauca (PgHOL1 and PgHOL2: DR569353 and TA16092_3330, respectively), Prunus persica (PpeHOL: TA5314_3760), Raphanus sativus (EW721469 and FD955532 for RsHOL1; EW738453 for RsHOL2), Selaginella moellendorffi (SmHOL: EFJ15331), Sorghum bicolor (SbHOL: Sb10g003780.1), Vitis vinifera (VvHOL1 and VvHOL2: GSVIVT01038247001 and GSVIVT01038246001, respectively), Zea mays (ZmHOL: TA188770_4577) were from the GenBank ${ }^{\mathrm{TM}}$ data base, The Arabidopsis Information Resource (TAIR; http://arabidopsis.org), The Rice Annotation Project Database (RAP-DB; http://rapdb.dna.affrc.go.jp), the Phytozome Database (http://www.phytozome.net), and the TIGR Plant Transcript Assemblies database (http://plantta.jcvi.org).

had 15 extra amino acids (GSTSLYKKAGSEFAL) at the $\mathrm{N}$-terminus of each OsHOL protein. Each recombinant protein was expressed in E. coli (BL21) and purified from the soluble fractions using Glutathione-Sepharose 4B (GE Healthcare) as described previously (Nagatoshi and Nakamura 2009). The purity and molecular weight of each recombinant protein were confirmed by SDSPAGE analysis (Figure 2A) and matrix-assisted laser desorption/ionization time-of-flight mass spectrometry (Autoflex Speed MALDI-TOF-MS; Bruker Daltonics, Bremen, Germany) (data not shown).

Using the purified recombinant OsHOL proteins, we then examined whether the proteins show SAMdependent synthesis of methyl iodide using iodide ions.
The assay mixture volume was $500 \mu \mathrm{l}$ and contained $0.5 \mathrm{mM}$ SAM (Sigma-Aldrich), $0.25 \mathrm{mM}$ KI (Wako Pure Chemical Industries), $0.1 \mathrm{M}$ Tris acetate (pH 7.5), and $2 \mu \mathrm{g}$ of OsHOL1 or OsHOL2 recombinant protein. Each of the assay mixtures and a standard methyl iodide (Nacalai Tesque) solution in 2-ml glass vials sealed with a screw cap fitted with a Teflonlined septum was incubated at $25^{\circ} \mathrm{C}$ for $1 \mathrm{~h}$ by shaking at $150 \mathrm{rpm}$. To the assay mixture, $1 \mathrm{M} \mathrm{HClO}_{4}(500 \mu \mathrm{l})$ was added to stop the reaction and the mixture was incubated at $70^{\circ} \mathrm{C}$ for $15 \mathrm{~min}$. Each sampled $100-\mu \mathrm{l}$ headspace was injected into the column of a gas chromatographymass spectrometer (GC-MS). GC/MS analyses were performed on an ion trap type GC-MS system (TRACE 


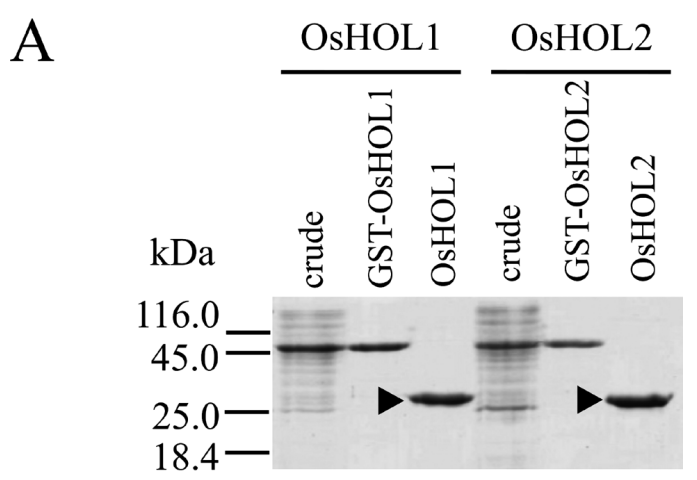

B
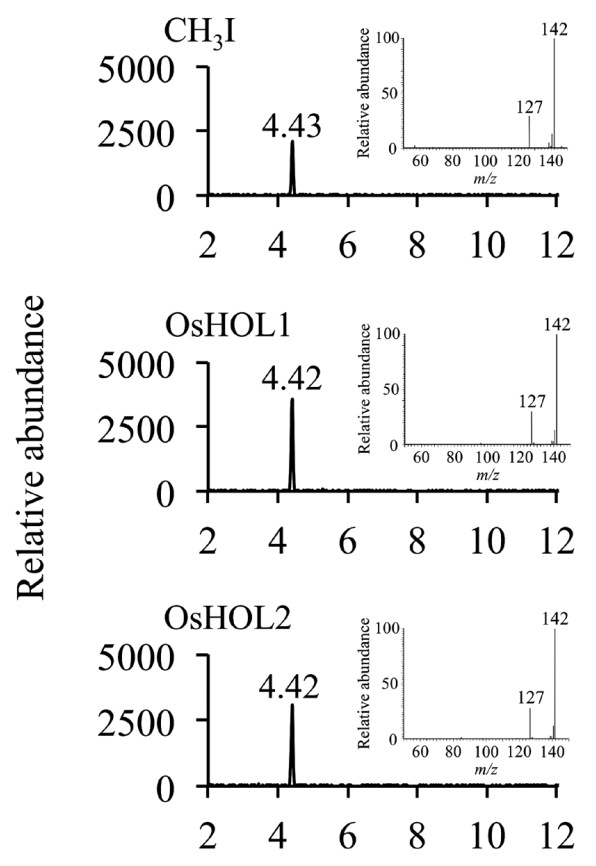

\section{Retention time (min)}

Figure 2. Characterization of recombinant OsHOL proteins. (A) SDS-PAGE of recombinant OsHOL proteins. Crude, soluble fraction of crude protein extracts; GST-OsHOL1and GST-OsHOL2, recombinant GST tagged OsHOL1and OsHOL2; OsHOL1 and OsHOL2, tagless OsHOL1 and OsHOL2. Arrowheads indicate the tagless OsHOL1 and OsHOL2. (B) GC/MS chromatograms for the standard methyl iodide, the reaction product of OsHOL1, and OsHOL2 monitored with SIM mode $(\mathrm{m} / \mathrm{z} 142)$. Insets show mass spectra at a retention time of $4.4 \mathrm{~min}$.

GC Ultra, ITQ900; Thermo Scientific) in electronionization (EI) mode $(70 \mathrm{eV})$. GC was performed on a DB-624 column $(0.25 \mathrm{~mm} \times 30 \mathrm{~m}, 1.4 \mu \mathrm{m}$ film thickness, Agilent) in splitless mode with helium as a carrier gas at a constant flow rate of $1 \mathrm{ml} \mathrm{min}^{-1}$. The injection port (splitless mode), interface, and MS source temperature were $200^{\circ} \mathrm{C}, 250^{\circ} \mathrm{C}$, and $200^{\circ} \mathrm{C}$, respectively. The GC temperature program began at $29^{\circ} \mathrm{C}(3 \mathrm{~min})$, was raised to $90^{\circ} \mathrm{C}$ at a rate of $8^{\circ} \mathrm{Cmin}^{-1}$, and was finally held at $90^{\circ} \mathrm{C}$ for $3 \mathrm{~min}$. GC/MS chromatograms and mass spectra were collected both in full scan mode $(\mathrm{m} / z 50-150)$ and in SIM mode $(\mathrm{m} / z$ 142). GC/MS analysis showed that both of the proteins produced chromatograms with peaks of the same retention time and mass spectra as standard methyl iodide (Figure 2B). These results indicated that OsHOL1 and OsHOL2 actually synthesized methyl iodide from SAM and an iodide ion.

Furthermore, we performed kinetic analysis of OsHOL proteins using halide and thiocyanate ions. SAM, KI, $\mathrm{KBr}, \mathrm{KCl}$ (Wako Pure Chemical Industries), and KSCN (Nacalai Tesque) were used as substrates. The assay mixture volume was $75 \mu \mathrm{l}$ and contained $0.1 \mathrm{M}$ Tris acetate ( $\mathrm{pH} 7.5), 1.5-5.0 \mu \mathrm{g}$ each of the recombinant OsHOL proteins and varying concentrations of each substrate. Each of the reaction mixtures was incubated at $25^{\circ} \mathrm{C}$ for $1-4 \mathrm{~h}$, determined beforehand to allow the reaction to proceed lineally. To stop the reaction, $1 \mathrm{M} \mathrm{HClO}_{4}(75 \mu \mathrm{l})$ was added to the assay mixture. After centrifugation $(16,100 \mathrm{~g}$ for $10 \mathrm{~min}), 50 \mu \mathrm{l}$ of the supernatant was injected into the HPLC system. The methyltransferase activities of OsHOL proteins were measured by quantifying the SAH produced by the enzymatic reactions. The HPLC analysis of SAH has been previously described (Nagatoshi and Nakamura 2009).

The kinetic parameters of rice HOL proteins were calculated for the first time in a non-Brassicales plant (Table 1). The methyltransferase activities of OsHOL proteins with chloride ions were below the detection limit under our assay condition. On the other hand, $E$. coli cells expressing OsHOL proteins were reported to release methyl chloride, although the synthesis level was much smaller than that of methyl iodide (Bayer et al. 2009). This discrepancy in methyl chloride synthesis will be due to differences between the assay conditions. Because rice tissues were reported to contain approximately $120 \mathrm{mmol} \mathrm{kg}^{-1} \mathrm{DW}$ of chlorine (Yuita 1983), these results suggested that the rice HOL proteins scarcely methylate chloride ion in vivo. The highest $k_{\text {cat }} / K_{\mathrm{m}}$ values were observed with iodide ions followed by thiocyanate ions for both of the HOL proteins. Because rice tissues contain neither glucosinolates nor cyanogenic compounds and produce few thiocyanate ions if any, a thiocyanate ion could not be a physiological substrate of the HOL proteins in rice. On the other hand, since the concentration of accumulated thiocyanate ions (ca. $140 \mu \mathrm{M}$ ) in homogenized Arabidopsis seedlings was much higher than the $K_{\mathrm{m}}$ value $(62 \mu \mathrm{M})$ for thiocyanate ions of the recombinant AtHOL1, a thiocyanate ion was suggested to be a physiological substrate in Arabidopsis (Nagatoshi and Nakamura 2009). The bromine content (approximately $150 \mu \mathrm{mol} \mathrm{kg}{ }^{-1} \mathrm{DW}$ ) was reported to be higher than the iodine content (approximately $5 \mu \mathrm{mol} \mathrm{kg}{ }^{-1} \mathrm{DW}$ ) in rice tissues (Yuita 1983). However, OsHOL1 and OsHOL2 showed $k_{\text {cat }} / K_{\mathrm{m}}$ values with iodide 
Table 1. Kinetic parameters for methyltransferase activities of recombinant OsHOL1 and OsHOL2

\begin{tabular}{|c|c|c|c|c|c|c|}
\hline & Variable substrate $(\mathrm{mM})$ & Isoform & $K_{\mathrm{m}}(\mathrm{mM})$ & $\begin{array}{c}\text { Vmax } \\
\left(\text { nmol s }^{-1} \text { mg protein }^{-1}\right)\end{array}$ & $k_{\text {cat }}\left(\mathrm{s}^{-1}\right)$ & $k_{\text {cat }} / K_{\mathrm{m}}\left(\mathrm{s}^{-1} \mathrm{mM}^{-1}\right)$ \\
\hline \multirow[t]{2}{*}{$\mathrm{KI}^{\mathrm{a}}$} & $3.13 \times 10^{-2}-5.00 \times 10^{-1}$ & OsHOL1 & $6.64 \times 10^{-2}$ & $8.65 \times 10^{-2}$ & $2.40 \times 10^{-3}$ & $3.62 \times 10^{-2}$ \\
\hline & $1.25 \times 10^{-1}-2$ & OsHOL2 & 1.14 & $3.43 \times 10^{-1}$ & $9.28 \times 10^{-3}$ & $8.11 \times 10^{-3}$ \\
\hline \multirow[t]{2}{*}{$\mathrm{KBr}^{\mathrm{a}}$} & $15-240$ & OsHOL1 & $4.46 \times 10^{1}$ & $5.65 \times 10^{-2}$ & $1.57 \times 10^{-3}$ & $3.52 \times 10^{-5}$ \\
\hline & $7.50-120$ & OsHOL2 & $3.37 \times 10^{1}$ & $2.60 \times 10^{-2}$ & $7.04 \times 10^{-4}$ & $2.09 \times 10^{-5}$ \\
\hline \multirow[t]{2}{*}{$\mathrm{KCl}^{\mathrm{a}}$} & $200-600$ & OsHOL1 & ND & ND & ND & ND \\
\hline & $200-600$ & OsHOL2 & ND & ND & ND & ND \\
\hline \multirow[t]{2}{*}{$\mathrm{KSCN}^{\mathrm{a}}$} & $5.00 \times 10^{-2}-8.00 \times 10^{-1}$ & OsHOL1 & $1.45 \times 10^{-1}$ & $1.61 \times 10^{-2}$ & $4.48 \times 10^{-4}$ & $3.08 \times 10^{-3}$ \\
\hline & $5.00 \times 10^{-2}-8.00 \times 10^{-1}$ & OsHOL2 & $2.46 \times 10^{-1}$ & $1.37 \times 10^{-2}$ & $3.72 \times 10^{-4}$ & $1.51 \times 10^{-3}$ \\
\hline \multirow[t]{2}{*}{$\mathrm{SAM}^{\mathrm{b}}$} & $6.25 \times 10^{-3}-1.00 \times 10^{-1}$ & OsHOL1 & $1.33 \times 10^{-2}$ & $5.56 \times 10^{-2}$ & $1.54 \times 10^{-3}$ & $1.16 \times 10^{-1}$ \\
\hline & $1.25 \times 10^{-2}-2.00 \times 10^{-1}$ & OsHOL2 & $2.97 \times 10^{-2}$ & $1.82 \times 10^{-1}$ & $4.92 \times 10^{-3}$ & $1.65 \times 10^{-1}$ \\
\hline
\end{tabular}

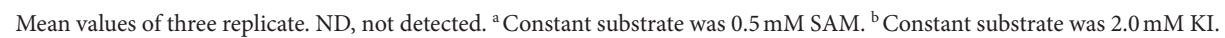

ions that were 1028- and 388-fold higher, respectively, than those with bromide ions. Therefore, when the kinetic parameters and halide concentrations in vivo are considered, both HOL proteins should mainly methylate iodide ions and not bromide ions.

Previous studies have shown methyl iodide emissions from rice paddies (Muramatsu and Yoshida 1995) and from rice tissues (Redeker et al. 2004). These phenomena could be because of enzymatic activity of OsHOL proteins in accordance with our biochemical results showing that both OsHOL1 and OsHOL2 actually have SAM-dependent methyltransferase activity toward iodide ions and synthesize methyl iodide. The widespread distribution of methyl iodide synthesis activity (Saini et al. 1995; Itoh et al. 2009) and HOL homologous proteins implied that iodide metabolism exists among land plants and HOL proteins play a role in it.

\section{Acknowledgements}

This work was supported by a Grant-in-Aid for Scientific Research (22780302) from the Ministry of Education, Science, Sports and Culture of Japan.

\section{References}

Attieh J, Djiana R, Koonjul P, Etienne C, Sparace SA, Saini HS (2002) Cloning and functional expression of two plant thiol methyltransferases: a new class of enzymes involved in the biosynthesis of sulfur volatiles. Plant Mol Biol 50: 511-521

Attieh J, Sparace SA, Saini HS (2000) Purification and properties of multiple isoforms of a novel thiol methyltransferase involved in the production of volatile sulfur compounds from Brassica oleracea. Arch Biochem Biophys 380: 257-266

Attieh JM, Hanson AD, Saini HS (1995) Purification and characterization of a novel methyltransferase responsible for biosynthesis of halomethanes and methanethiol in Brassica oleracea. J Biol Chem 270: 9250-9257

Bayer TS, Widmaier DM, Temme K, Mirsky EA, Santi DV, Voigt CA (2009) Synthesis of methyl halides from biomass using engineered microbes. J Am Chem Soc 131: 6508-6515

de Benoist B, McLean E, Andersson M, Rogers L (2008) Iodine deficiency in 2007: global progress since 2003. Food Nutr Bull 29:
195-202

FAOSTAT (2010) Food and Agricultural Organization of the United Nations. FAOSTAT Statistical Database (accessed Jan 8, 2012)

Horton P, Park K-J, Obayashi T, Fujita N, Harada H, Adams-Collier CJ, Nakai K (2007) WoLF PSORT: protein localization predictor. Nucleic Acids Res 35(Web Server issue): W585-7

Itoh N, Toda H, Matsuda M, Negishi T, Taniguchi T, Ohsawa N (2009) Involvement of $S$-adenosylmethionine-dependent halide/ thiol methyltransferase (HTMT) in methyl halide emissions from agricultural plants: isolation and characterization of an HTMT-coding gene from Raphanus sativus (daikon radish). BMC Plant Biol 9: 116

Lassmann T, Sonnhammer ELL (2005) Kalign—an accurate and fast multiple sequence alignment algorithm. BMC Bioinformatics 6: 298

Mayer JE, Pfeiffer WH, Beyer P (2008) Biofortified crops to alleviate micronutrient malnutrition. Curr Opin Plant Biol 11: $166-170$

Midorikawa K, Nagatoshi Y, Nakamura T (2009) A selection system for transgenic Arabidopsis thaliana using potassium thiocyanate as the selective agent and AtHOL1 as the selective marker. Plant Biotechnol 26: 341-344

Muramatsu Y, Yoshida S (1995) Volatilization of methyl iodide from the soil-plant system. Atmos Environ 29: 21-25

Nagatoshi Y, Nakamura T (2009) Arabidopsis HARMLESS TO OZONE LAYER protein methylates a glucosinolate breakdown product and functions in resistance to Pseudomonas syringae pv. maculicola. J Biol Chem 284: 19301-19309

Nagatoshi Y, Nakamura T (2007) Characterization of three halide methyltransferases in Arabidopsis thaliana. Plant Biotechnol 24: 503-506

Ni X, Hager LP (1998) cDNA cloning of Batis maritima methyl chloride transferase and purification of the enzyme. Proc Natl Acad Sci USA 95: 12866-12871

Ni X, Hager LP (1999) Expression of Batis maritima methyl chloride transferase in Escherichia coli. Proc Natl Acad Sci USA 96: 3611-3615

Ohsawa N, Tsujita M, Morikawa S, Itoh N (2001) Purification and characterization of a monohalomethane-producing enzyme $S$ adenosyl-L-methionine: halide ion methyltransferase from a marine microalga, Pavlova pinguis. Biosci Biotechnol Biochem 65: 2397-2404

Redeker KR, Manley SL, Walser M, Cicerone RJ (2004) Physiological and biochemical controls over methyl halide 
emissions from rice plants. Global Biogeochem Cycles 18: GB1007

Rhew RC, Østergaard L, Saltzman ES, Yanofsky MF (2003) Genetic control of methyl halide production in Arabidopsis. Curr Biol 13: 1809-1813

Saini HS, Attieh JM, Hanson AD (1995) Biosynthesis of halomethanes and methanethiol by higher plants via a novel methyltransferase reaction. Plant Cell Environ 18: 1027-1033

Sato Y, Antonio BA, Namiki N, Takehisa H, Minami H, Kamatsuki K, Sugimoto K, Shimizu Y, Hirochika H, Nagamura Y (2011) RiceXPro: a platform for monitoring gene expression in japonica rice grown under natural field conditions. Nucleic Acids Res 39(Database issue): D1141-D1148

Saxena D, Aouad S, Attieh J, Saini HS (1998) Biochemical characterization of chloromethane emission from the wood- rotting fungus Phellinus pomaceus. Appl Environ Microbiol 64: 2831-2835

Toda H, Itoh N (2011) Isolation and characterization of a gene encoding a $S$-adenosyl-L-methionine-dependent halide/ thiol methyltransferase (HTMT) from the marine diatom Phaeodactylum tricornutum: Biogenic mechanism of $\mathrm{CH}_{3} \mathrm{I}$ emissions in oceans. Phytochemistry 72: 337-343

Yuita K (1983) Iodine, bromine and chlorine contents in soils and plants of Japan : III. Iodine, bromine and chlorine contents in the andosols and in plants of central Honshu. Soil Sci Plant Nutr 29: 403-428

Zimmermann MB, Jooste PL, Pandav CS (2008) Iodine-deficiency disorders. Lancet 372: 1251-1262 